\title{
Visual stimuli on the Commodore Amiga: A tutorial
}

\author{
STUART ANSTIS \\ York University, Downsview, Ontario, Canada
}

\begin{abstract}
The Commodore Amiga home microcomputer, together with DeLuxePaint, a commercial software package, can generate many useful visual stimuli, including random-dot stereograms, apparent motion, texture edges, aftereffects from dimming and brightening, motion aftereffects, dynamic random noise, and drifting and counterphase gratings. Videotapes can readily be made of these displays. No programming experience is necessary.
\end{abstract}

For many visual scientists, the modern microcomputer has replaced a whole roomful of more specialized stimulus-generating equipment (Cavanagh \& Anstis, 1980). The Commodore Amiga has without doubt the best graphics of any current home computer, and DeLuxePaint, by Daniel Silva (Electronic Arts, 1820 Gateway Drive, San Mateo, CA 94404) is one of the best graphics programs available. It is like MacPaint on the Macintosh plus color plus look-up tables plus additional features. The program requires no programming skills at all; the user draws directly on the screen by moving a mouse on the table top. This article shows the reader how to produce some commonly desired visual stimuli by means of the DeLuxePaint program. It is assumed that the reader has mastered the 32-page manual that comes with the program.

\section{Random-Dot Stereogram}

First make a random-dot texture and make two identical copies of it side by side, one for each eye, then introduce disparity, either in the form of a central disparate patch or by curving the whole curved area in depth.

To make the random-dot texture, start by clearing the screen to white. Select the 1-pixel brush and the airbrush tool, with black as the foregound color. Now "scribble" with the mouse, moving it very fast, so that it lays down an irregular spray of black dots. Clean things up by adding black and white dots by hand in the magnify mode until you are satisfied with the pseudorandom dot texture. Turn magnify off.

To duplicate the texture, pick up a tall rectangle of texture about $7 \mathrm{~cm}$ wide and $10 \mathrm{~cm}$ high with the brush selection tool. Clear screen to white (this will not destroy the dot texture you just picked up as a brush). Print down two identical copies of the brush rectangle side by side, one for each eye.

To introduce disparity, you can select two types of depth pattern: a central square region that stands out in front

This work was supported by Grant A 0260 from the Natural Sciences and Engineering Research Council of Canada. Reprint requests should be sent to Stuart Anstis, Department of Psychology, York University, 4700 Keele St., Downsview, Ontario, Canada M3J 1 P3. of a back surround plane, or a single surface that is curved in depth.

To produce a square region floating above a flat background (Julesz, 1971), set the background color to nonwhite, to avoid transparencies in the brush you are about to pick up. Pick up a square region in the center of one of the two rectangles, say the right one. (This does not leave a hole; the brush copies the picked-up region without deleting it.) Move it a few millimeters to the left, and print it down again. If you now free-fuse the two rectangles (Figure 1a) by diverging your eyes, you will see the central area, which has a few millimeters of crossed disparity, as a square floating in depth above the background. If you free-fuse by converging your eyes, the central square will look like a window to a texture lying behind the plane of the screen.

To produce a single surface that is curved in depth, skip the instructions in the preceding paragraph. Instead, select the whole of the right eye's rectangle with the brush selection tool. Select "Bend-Horizontal" from the "Brush" menu. Now bend the right eye's rectangle so that the central part halfway down the rectangle bows out to the left like a shallow letter $\mathrm{C}$. If you now free-fuse this bent rectangle seen by the right eye with the straight rectangle seen by the left eye (Figure 1b), the whole rectangle will appear curved in depth, with the central part farther away than the top and bottom (or nearer, depending on whether you converge or diverge when you free-fuse).

To make a stereoscopic grating (Tyler, 1974), start off with a short, wide random-dot rectangle about $5 \mathrm{~cm}$ wide and $1 \mathrm{~cm}$ high. As before, print two identical copies side by side, one for each eye, and bend the right eye's rectangle horizontally. When the two rectangles are fused, the disparity will make the resulting figure look rather like a convex round horizontal rod. Now select the right eye's bent rectangle with the brush selection tool. Hit $\mathrm{X}$ to reverse it left to right. Print it just below but touching the original bent rectangle seen by the right eye. In the same way, pick up the unbent rectangle seen by the left eye, hit $\mathrm{x}$ to reverse it left to right, and print it just below but touching the original unbent rectangle seen by the left eye. When the four rectangles are fused, you will see a 

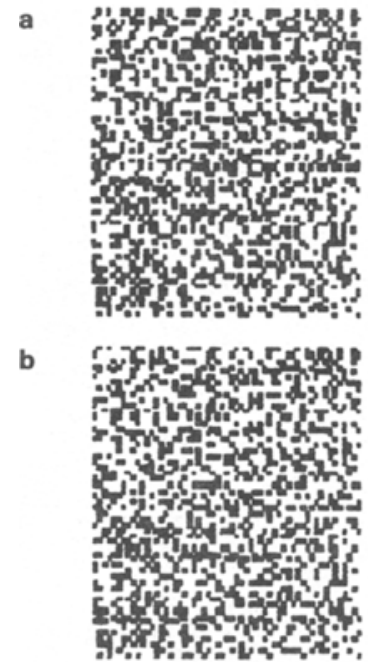

c

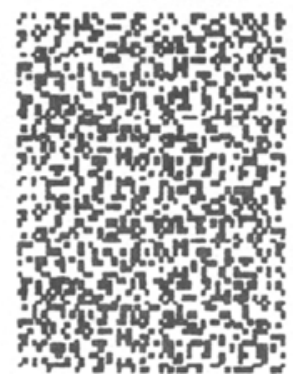

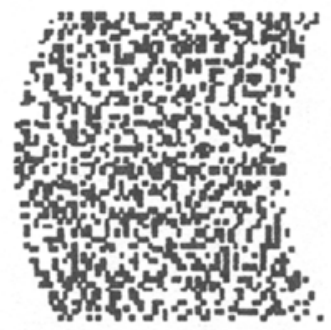
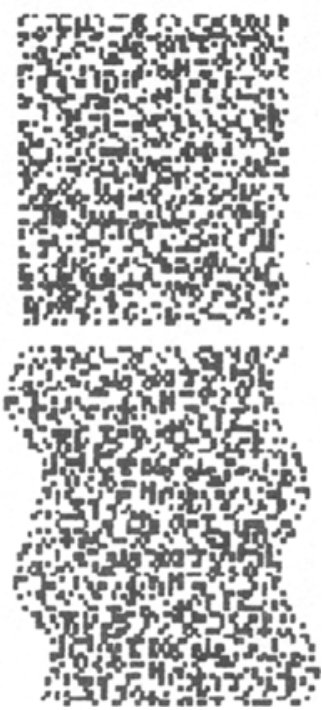

Figure 1. Random-dot stereograms (Julesz, 1971). (a) Right eye's picture was bent by selecting the "Brush-Bend horizontal" menu. When fused, a surface is seen that is curved in depth. (b) A central region in the right eye's picture was shifted sideways by a few millimeters. When fused divergently, this central region appears to lie in front of the surround (or behind, if convergent free fusion is used). (c) A stereoscopic grating (Tyler, 1974), produced by the same technique as (a).

corrugated surface bent into an $S$ in depth and comprising a single cycle of a stereoscopic grating with horizontal bars (Figure 1c). Select this whole binocular pattern as a brush and print it below itself to add one more cycle to the stereo grating, and continue until you have enough cycles. To make a twinkling dynamic random-dot stereogram, see the Dynamic Random-Dot Texture section below.

\section{Apparent Motion With Color Cycling}

Apparent motion can be produced by cycling the colors via the Amiga's built-in look-up tables.

In the default palette the first three colors are black, white, and red. Clear the screen to black. Put a white spot in the left half of the screen and a red spot in the right half. Now hit $p$ to go to the palette in order to set up a cycle range. You can have up to three different cycle ranges for each picture. Click $\mathrm{C} 1$ to select the range you want to change. Click on color \#2 (white), then on RANGE, then on color \#3 (red). The bracket to the left of the colors shows which colors are currently included in the selected range. To control the cycle speed for the selected range, set the speed bar on the palette to about halfway along.

Hit OK to exit from the palette, and hit TAB to start the color cycle. Each spot will alternate in color between red and white; first the left spot will be white and the right spot red, then the left spot will be red and the right spot white (Figure 2a). To convert this alternation into apparent motion, use a hide-and-reveal technique: hit $p$ again to return to the palette, and change color \#3 from red to black by moving the palette slider marked $\mathrm{V}$ (for Value) down as far as it will go. Once again, hit OK to exit from the palette, and hit TAB to start the color cycle. Each spot will now alternate between black and white. First you will see a white spot on the left; the right spot, being the same black as the background, is invisible. Then you will see a white spot on the right; the left spot, being the same black as the background, is invisible. The result is a white spot that jumps back and forth between two positions (Figure 2b).

To make a spot jump along through five positions, reinstate the "Default Palette" under "Color Control"' in the "Picture" menu. Clear the screen to black, and put a row of five spots in colors \#2 through \#6 (white, red, brown, buff, yellow) across the screen. Go to the palette, click $\mathrm{C} 1$, then color \#2 (white), then RANGE, then color \#6 (yellow). Leave color $\# 2$ as white, but change all the colors \#3 through \#6 to black by selecting each color in turn and moving the V slider all the way down. Exit from the palette and hit TAB. You will see a white spot jumping repetitively through five positions across the screen.

A nice example of apparent motion appears on the demonstration picture "Warpresstab" that comes with the Amiga. A starship is seen hanging amid stars that stream continuously outward from a central point. To see how

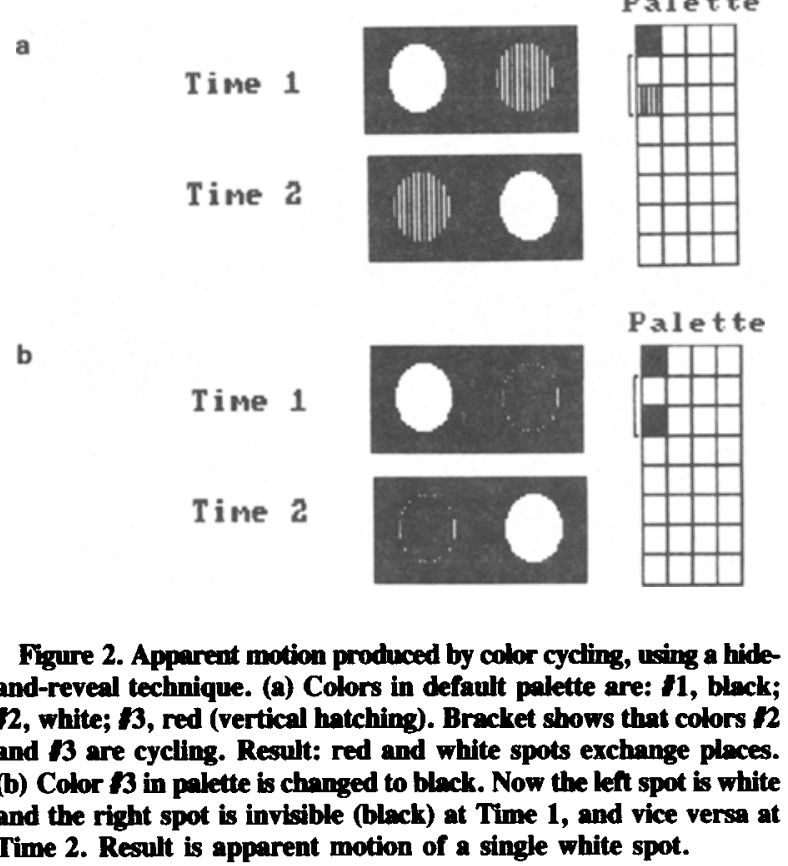

Figure 2. Apparent motion produced by color cycling, using a hideand-reveal technique. (a) Colors in default palette are: $\mathbf{H}$, black; $\boldsymbol{n}$, white; $\boldsymbol{A 3}$, red (vertical hatching). Bracket shows that colors $\boldsymbol{n}$ and $\boldsymbol{B}$ are cycling. Result: red and white spots exchange places. Time 2. Result is apparent motion of a single white spot. 
the artist achieved this effect, select the "Default Palette" from the "Picture-Color Control" menu. You will see that the radial trajectory of each star consists of a radial row of dots whose colors are $\# 1, \# 2, \# 3, \ldots$ in the palette. Select the "Restore Palette" from the "Picture-Color Control" menu, and color \#1 will become white and the other colors black. Hit TAB to start the color cycle, and dot \#1 will briefly turn white, then dot \#2, then dot \#3, and so on, all the other dots being black. Thus one white dot will be seen to jump successively through positions $\# 1, \# 2, \# 3, \ldots$ on a black background.

An entirely different but limited way of producing apparent motion is to draw two different pictures on the two graphics pages, then switch back and forth between them by hitting j. (Make sure that the CAPS LOCK key is not depressed). Draw the first picture, say a red circle in the left half of the screen. Hit $j$ to expose the spare screen. Draw a second picture-say a blue square-in the right half of the screen. Now each time you hit $j$ you will toggle from one screen to the other. Tapping on $\mathrm{j}$ repeatedly will reveal a circle that jumps across, changing into a square, and jumps back again.

\section{Areas Filled With Texture}

A white square on a black surround is defined by its luminance. A square of fine random-dot texture may have the same space-averaged luminance as a coarse randomdot background texture, but is visible because it is defined by the difference in texture. What texture differences the eye can detect is an empirical question, and stimuli to investigate this can be generated readily with DeLuxePaint. A 512K Amiga has two display pages available, which are swapped by hitting $j$ on the keyboard. (A 128K Amiga does not have the second page.)

Clear both display pages to black. Cover one page with a fine texture, say by spattering it with small red dots, and cover the other page with a coarse texture, say by spattering it with larger green dots. Draw a yellow square in the middle of the coarse texture. Now select this yellow as the background color. Go to the "Picture" menu, select the "Spare" heading and the "Merge in back" subheading. The yellow color will burn through to the back page, revealing a square region of fine texture.

This technique can be used to define an optical illusion with vernier (phase) edges (Figure 3c). Fill the whole of one page with a grating of horizontal lines; select the grid tool with the right-hand button on the mouse and set the grid separation to $2 \times 2$ pixels. With the 1 -pixel brush and the straight-line tool, draw a grating of horizontal lines. The grid will constrain the separation to 2 pixels. Turn off the grid and pick up the whole page with the brush selection tool. Move it 1 pixel vertically so that it is in spatial counterphase with the grating on the screen (Figures 3a, 3b). Change to the second page by hitting $\mathrm{j}$, and click the left button to print the phase-shifted grating on the second page. De-select the grating as brush by selecting the 3-pixel brush and the straight-line tool. Hit $\mathbf{j}$ a couple of times to confirm that the grating jumps up and down as you swap pages. Now paint a Müller-

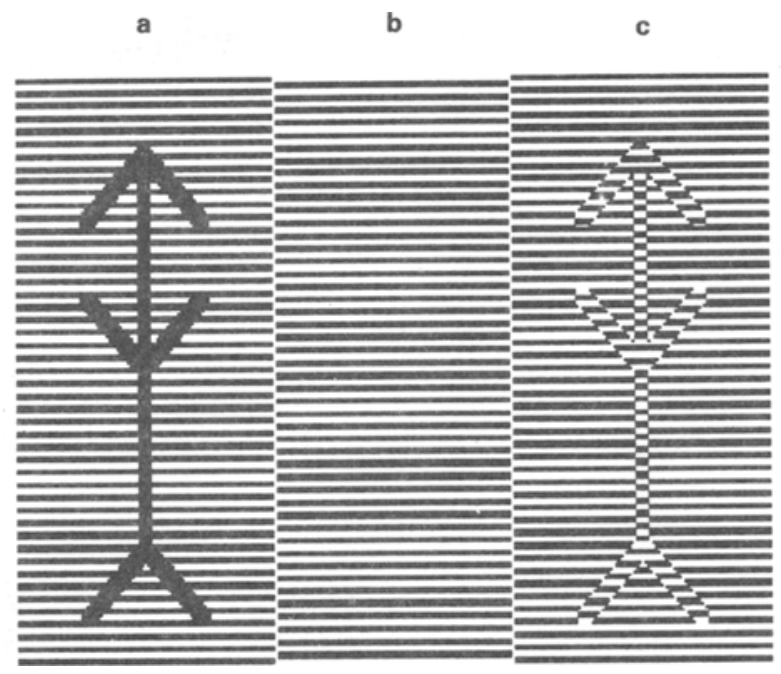

Figure 3. Müller-Lyer figure defined by the vernier or phase edges between two striped areas. (a) Page 1, with the Miller-Lyer figure drawn in the current background figure on a background of parallel lines. (b) Page 2, filled with horizontal lines that do not line up with those on Page 1. (c) Results of selecting the "Picture-Spare-Merge in back" menu while viewing Page 1; the arrow-shaped region of background color burns through to reveal the phase-shifted lines of Page 2.

Lyer figure, with its long axis vertical, in the current background color, say yellow. Select "Merge in back" from the "Picture-Spare" menu. The yellow will burn through to the back page, revealing a Müller-Lyer figure defined by phase edges (Figure 3c). This figure contains no spatial frequencies lower than that of the grating. Confirm this by putting tracing paper over the screen: the figure vanishes. This demonstrates that the Müller-Lyer effect is not dependent on the presence of low spatial frequencies.

\section{Aftereffects From Brightening and Dimming}

Following adaptation to a uniform field that is gradually brightening, a steady test field appears to be gradually dimming, and vice versa (Anstis, 1967, 1986). This phenomenon can easily be demonstrated on the Amiga.

Select the default palette from the "Picture-Color Control" menu. (The default palette always appears when you first switch on, without your needing to select it.) Notice that the 32 colors in the palette are arranged in four columns with 8 colors per column. We shall refer to the colors in the first column as \#1 through \#8, those in the second column as \#9 through \#16, and so on.

Clear the screen to green \#8-the bottom color in the first column of the palette. Notice that the last 12 colors on the palette form a graded series of grays, from nearblack to near-white. Draw a filled square about $8 \mathrm{~cm}$ across using color \#25 (the gray that is the top color in the fourth [right-hand] column of the palette). Put a red fixation dot in the center. Hit $p$ to select the palette, and define a color cycle in the palette from near-black ( $\$ 21$, fifth color in the third column) to near-white (\#32, bot- 
tom color in the fourth column). To define this color cycle, click in turn on the following palette locations: $\mathrm{Cl}$, color \#21, RANGE, color \#32. A white vertical bracket will appear, extending between colors $\# 21$ and $\# 32$. The horizontal speed bar in the palette contains nine speed markers, the ninth marker on the right being the fastest. These form a logarithmic series, with each marker producing half the exposure time of its neighbor. Thus, when $\mathrm{C} 1$ is selected and $\mathrm{C} 2$ and $\mathrm{C} 3$ are disabled, the approximate durations of the colors in the $\mathrm{C} 1$ range are $1,2,4,8$, $16,32,64,128$, and 256 TV frames when the speed control is progressively moved from the ninth (fastest) marker on the right, down through the eighth, seventh, sixth, and so on, to the first (slowest) marker on the left.

Select the eighth marker. Click on OK to close the palette and return to the screen.

Now hit the TAB key to make the colors cycle. The luminance of the central square will now be ramp modulated over time, repeatedly growing dimmer and then brightening abruptly. Fixate the red dot and adapt for about $30 \mathrm{sec}$. Hit TAB again to stop the cycle, and you will see an aftereffect in which the central square will appear to be gradually brightening (Anstis, 1967). To add a second square, which repeatedly brightens while the first square is dimming (Figure 4), redefine the palette as follows. Set color \#5 in the first column to white, and color \#16 (bottom color in the second column) to black. Use the "Spread" function in the palette to set up a graded series of grays from white to black between colors \#5 and $\# 16$, and set up cycle $\mathrm{C} 2$ in the palette to cycle through these colors. Draw a filled square in color \$9 (top color in the second column). Hit TAB, and one square will brighten while the other dims. Adapt to these, then hit TAB, and two opposite aftereffects of brightening and dimming will be seen, one above the other.

a

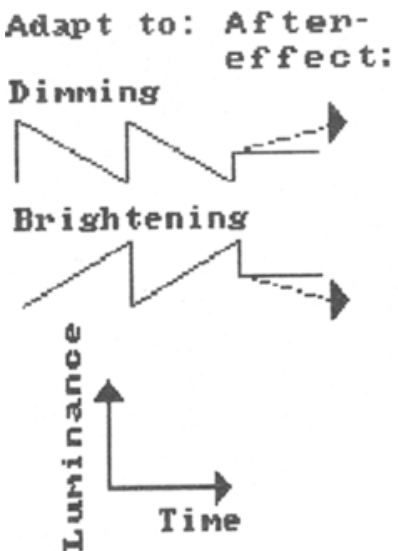

Figure 4. Aftereffect produced by dimming and brightening patches. (a) Time-luminance diagram: the adapting stimuli are ramp modulated and the test stimuli are steady in luminance (solid lines). During the aftereffects (dashed arrows) the upper, dimming, stimulus appears to brighten, and the lower, brightening, stimulus appears to grow dimmer. (b) Stimuli are gray patches. (c) Palette colors.

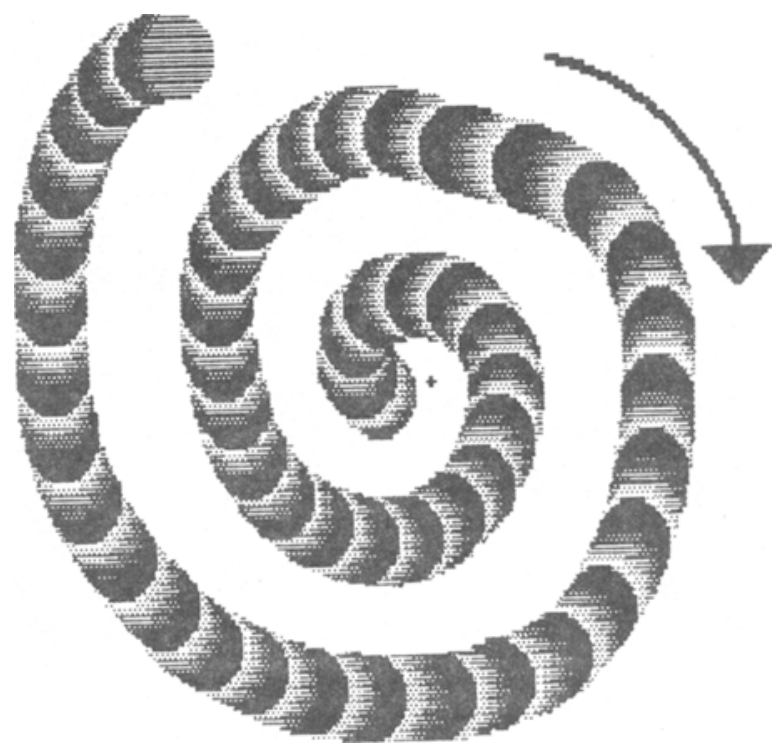

Figure 5. Motion aftereffect. Spirol, drawn in cycle mode, appears to move clackwise (arrow) when TAB is pressed. Following 30 sec adaptation, pressing TAB again will halt the motion and produce a negative (counterclockwise) aftereffect of motion.

\section{Motion Aftereffect}

Keep the same color cycles as before, but increase the speed to maximum by pushing all the speed bars in the palette all the way to the right. Select the larger diskshaped brush with the right-hand button on the mouse, and enlarge it to about $1 \mathrm{~cm}$ in diameter. Pick color $\$ 25$ (top color in the fourth column) as the foreground color, and from the "Color (Mode)" menu select "Cycle." Now that "Cycle" is in effect, the line will not be uniformly gray but will be banded transversely like a snake (Figure 5). Put a red fixation dot in the middle of the screen. Hit the TAB key to start the color cycle, and the transverse gray bands will be seen running continuously clockwise along the spiral, as indicated by the arrow in Figure 5. Adapt to the motion by fixating the central spot for about $30 \mathrm{sec}$, then hit TAB again to stop the motion. A strong negative aftereffect of motion will be seen.

\section{Dynamic Random-Dot Texture}

A dynamic snowstorm of twinkling random dots, looking like the output of a detuned television receiver, is a useful tool in vision research (Mackay, 1965). Keep the same color cycle for the gray colors $\# 21-\# 32$ as in the previous example. Clear the screen to black. In magnify mode with the 1-pixel brush and the dotted-line tool, plot a tiny $6 \times 4$ checkerboard in red. Each square of the checkerboard is only 1 pixel in size. Make six copies of the checkerboard with the brush selection tool (Figure 6). Pick up each gray color in turn and fill two 1-pixel squares, chosen at random, of the first checkerboard (you are filling 24 pixels with 12 colors). Do the same for each checkerboard, choosing two different random squares each time. It is a good idea to fill in the black pixels on 


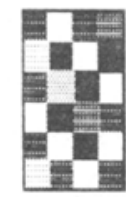

A

b

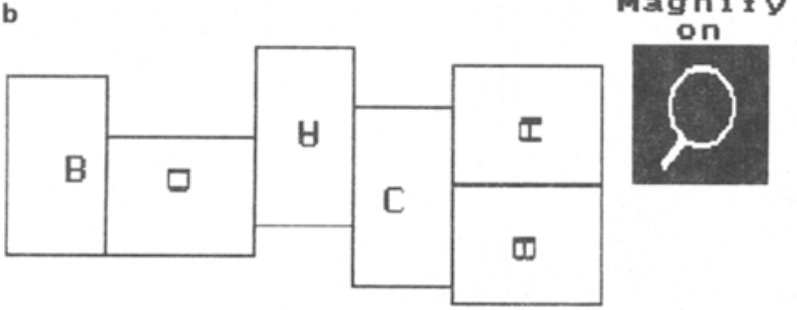

Figure 6. Plotting pseudorandom dynamic visual noise. (a) In magnify mode, tiny matrices or checkerboards of one-pixel checkerboards are drawn (C, D) and filled with pseudorandom gray levels (A, B). (b) These matrices are randomly reflected and rotated, then glued together in random orientations. See text.

each checkerboard with the six darker grays and the red pixels with the six lighter grays. When you have finished you have six checkerboards filled with pseudorandom grays, looking like little pieces of sandpaper. Copy your six checkerboards to fresh areas of the screen, after mirror-reversing them horizontally in the $x$ axis or vertically in the $y$ axis (by hitting $\mathrm{x}$ or $\mathrm{y}$ on the keyboard) and/or rotating them through one or more right angles (by hitting $\mathrm{z}$ one or more times). Each checkerboard has eight possible combinations of reflection and rotation. Use the brush selection tool to pick up different $6 \times 4$ checkerboards and agglomerate them into a larger checkerboard (Figure 6b). Turn off the magnify mode. When you have a reasonably large agglomeration, pick that up in turn with the brush selection tool and copy it, after reflections and rotations, to a different part of the screen. Now glue these agglomerates together until most or all of the screen is covered with a static pseudorandom-dot texture containing 12 gray levels. If you wish, you can hit $\mathrm{p}$ to select the palette and randomly EXchange the positions of the 12 grays within the palette.

Hit the TAB key, and there is your dynamic twinkling texture. To make a dynamic random-dot stereogram, pick up a rectangular region of texture and follow the procedures given in the Random-Dot Stereogram section above.

\section{Making a Drifting Grating}

The palette consists of 32 colors, and in the default palette the last 12 of these ( $\$ 21$ through $\# 32$ ) are a graded set of grays. First draw a spatial gradient consisting of a line of 12 pixels, each colored a different gray. Then convert this gradient into a grating.

Select the 1-pixel brush, the straight-line tool, and color \#21 (dark gray). Switch on the magnify mode and draw a single dark-gray pixel. Advance one color (to $\$ 22$, a slightly lighter gray) by hitting the ] key with your left hand. Draw a second pixel to the right of but touching the first. Continue this process until you have a horizontal line of 12 pixels, spatially shaded from dark (color \#21) on the left to light (color \#32) on the right. Turn off the magnify mode. Extend this line up and down the full height of the screen by selecting it with the brush selection tool, holding down the shift key, and moving the brush up and down through the full height of the screen. Now select this long shaded bar with the brush selection tool and print copies of it, side by side and touching, across the full width of the screen. You now have a grating with a ramp or sawtooth luminance profile.

To drift the grating, hit $\mathrm{p}$ to go to the palette and cycle colors \#21 through \#32. Hit the TAB key to start the color cycle running.

It is easy to alter the luminance profile of the grating by changing the gray levels in the palette. To make a square wave grating, make colors $\$ 21$ through $\$ 26$ black, and make colors \#27 through \#32 white (Figure 7). To give the grating a triangular waveform, which will look not unlike a sinusoidal grating, make the color $\$ 21$ black and color \#26 white, then use the "Spread" function in the palette to produce a graded series of grays between them. In the same way, make color $\$ 27$ white and color \#32 black, and make a graded series of grays between them. Once you have redefined the luminance profile, the grating will drift when you hit the TAB key.

You can superimpose two gratings that drift in opposite directions by plotting one grating on the oddnumbered horizontal lines of the TV raster and the other grating on the even-numbered lines. If the two gratings, one drifting to the left and the other to the right, are identical, the outcome is a counterphase flickering grating. To make a counterphase grating, clear the screen and repeat the initial stage of making an ordinary grating; that is, turn on the magnify mode and plot a horizontal row of single pixels ranging in color from $\$ 21$ (dark gray) on the left to \#32 (light gray) on the right. Now pick this up with the brush selection tool, hit $x$ to reverse it left to right, and print it underneath but touching the previous horizontal line (Figure 7d). Pick up this double row and print it underneath but touching itself. Do this many times until you have a column the height of the screen, composed of layers in which the odd-numbered horizontal lines go from $\# 21$ to $\# 32$ and the even numbered lines go from \#32 to \#21. Now select this long column with the brush selection tool and print copies of it, side by side and touching, across the full width of the screen. You now have two superimposed ramp or sawtooth gratings. Hit the TAB key to start the color cycle running (you already selected a color cycle from $\# 21$ to $\# 32$ in the palette). The two ramp gratings will drift in opposite directions. Hit TAB to stop the color cycle, hit $p$ to go to the palette, and reset colors \#21 through \#32 to give a sinusoidal luminance profile. Hit $O K$ to exit from the palette, hit TAB to restart the color cycle, and there is your counterphase flickering grating. A sheet of tracing paper taped to the 
a

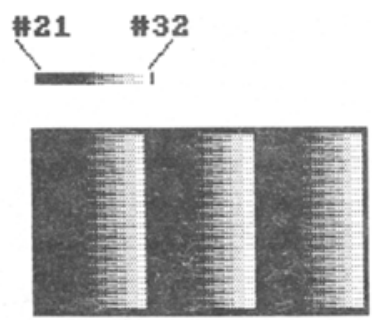

b
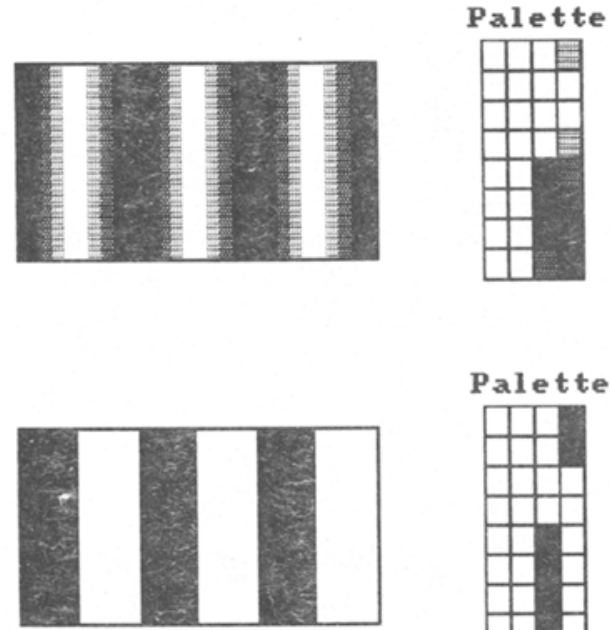

Palette

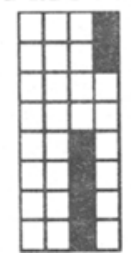

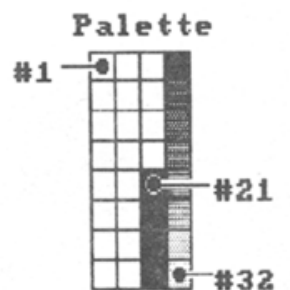

d

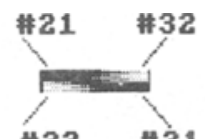

\#32

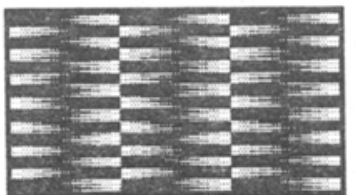

来1

Palette
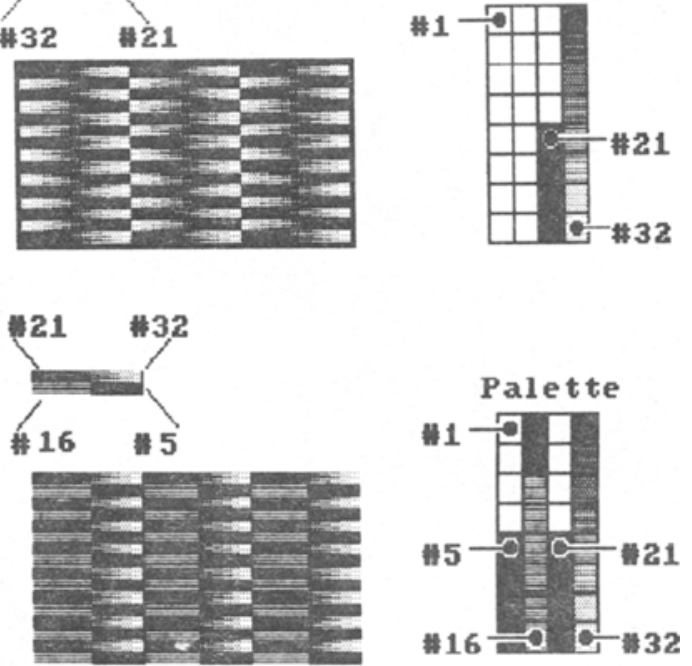

f

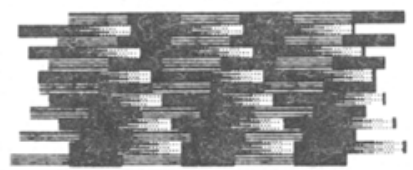

Figure 7. Plotting a grating. (a) In magnify mode, a shaded horizontal line is drawn comprising 12 adjacent pixels in colors 21 through 132. The line is smeared vertically into a bar, then the bar is replicated three times to form a smoll grating. These colors are shown in the defaalt palette at right. Luminance profile of (a) is changed into a triangular (b) or square wave (c) grating, simply by changing the palette colors. (d) Counterphase grating: In magnify mode, a 12-pirel horizontal line is drawn in colors $\mathbf{2 1}$ through $\mathbf{3 2}$, as in (a). It is then picked up, reversed left to right by pressing $x$, and printed below itself. The double row is copied vertically into a vertical bar like a sandwich, then the bar is replicated three times to form a small grating. (e) Two different gratings drifting in oppostte directions: a 12-pixel line is drawn in colors $\mathbf{2 1}$ through $\mathbf{3 2}$, as in (a) and (d). For the second grating a 12-pixel line is drawn below it in colors 16 through $\mathbf{5}$. The double row is then replicated as in (a) and (d). (f) To tilt the two gratings shown in (e), the horizontal lines are displaced sideways during replication.

screen will eliminate any unwanted raster detail. If you want to superimpose two different gratings drifting in opposite directions, plot a horizontal line of pixels in colors \#21 through \#32, as before. Below this line and touching it, plot a line of pixels to define the second grating, using colors \#16 through \#5 (not \#5 through \#16, because you want this grating to drift in the opposite direction) (Figure 7e). Make multiple copies of this double line to fill the entire screen, as before. Then go to the palette and put the desired luminance profile for the second grating into colors \#5 through \#16. To produce gratings that are tilted away from the vertical, misalign or offset the pixel lines horizontally (Figure 7f).

To have different areas of drifting gratings on the screen at the same time (Figure 8), select a part of the grating with the brush selection tool, modify it by resizing, reflecting, rotating, or shearing it via the "Brush" menu, and print this patch of modified grating onto the screen. (You may find that some brush transformations distort the original grating unacceptably.) For instance, suppose you want a circular window of horizontal grating centered in a screen that is filled with a vertical grating. Start with the vertical grating and select a large square patch of it with the brush selection tool. Hit $\mathrm{z}$ to rotate this patch through a right angle. Hit $j$ to go to the second screen. Print down the horizontal grating, which is your current brush, into the center of the second screen. Hit $j$ to return to the first screen and draw a filled circle in the middle of the screen in the current background color (any color will do, provided it does not appear anywhere else on the screen). From the "Picture" menu select "Spare-Merge in back." The current background color will burn through to the second screen to reveal a circular patch of horizontal grating. Hit $T A B$ to make both gratings drift.

\section{Making a Videotape}

If you wish to demonstrate your computer pictures at a lecture or conference, it is easy to videotape the display. Connect an RF cable with an RCA phono plug at each end from the (composite) video output on the back of the Amiga to the video input socket on the back of a videocassette recorder (VCR). An RF cable delivers bet- 


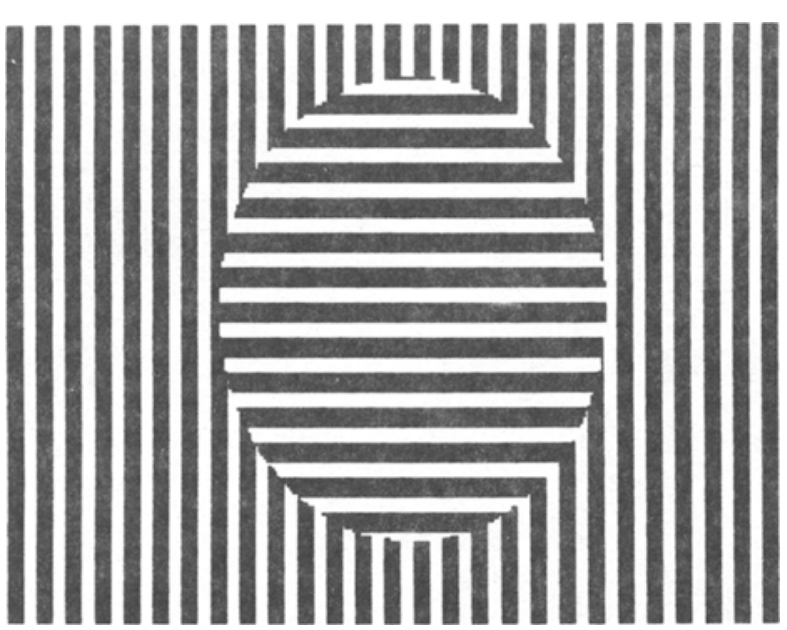

Figure 8. A circular window filled with one grating is centered in a surround of a second drifting or stationary grating at a different orientation. See text.

ter image quality than an audio cable. Load your program, animate it by hitting TAB if necessary, then hit the record button on the VCR. If you record a series of programs, hitting the stop button on the VCR between programs, there will be an undesirable 3-sec gap on the videotape between programs. To avoid this, hit the pause button instead of the stop button on the VCR after recording a program, load the next program, and hit the record button. Do not leave the pause button depressed for any longer than necessary since it is apt to wear a hole in the tape. Some VCRs turn off automatically after $5 \mathrm{~min}$ if left on pause.

\section{Importing Pictures Into a BASIC Program}

A picture drawn by a paint program can be called up from within a BASIC program. (Such a program might display a sequence of pictures to a subject and collect his/her responses to each one.) The conventions for importing pictures, called Interleaved File Format or IFF, will be published in the next revision of the Amiga ROM Kernel Manual.

\section{Other Paint Programs}

Two other paint programs are currently on the market. Commodore's Graphicraft program is less versatile for psychophysics than is DeLuxePaint. A new contender is Aegis Images, which resembles DeLuxePaint and comes packaged with the remarkable Aegis Animator, which allows the user to make animated movies (Aegis Development, Inc., 2210 Wilshire Boulevard, Suite 227, Santa Monica, CA 90403). The user of Animator draws a series of "key" pictures, such as a triangle at the top of the screen followed by a square at the bottom of the screen. The program automatically interpolates pictures in between, as an animation fill-in artist would do, so that on the RUN command a triangle is seen moving at any desired speed down the screen, gradually changing into a square as it moves.

\section{REFERENCES}

ANSTIS, S. M. (1967). Visual adaptation to gradual change of luminance. Science, 155, 710-712.

ANSTIS, S. M. (1986). Recovering movement information from luminance. Vision Research, 26, 147-160.

Cavanagh, P., \& Anstis, S. M. (1980). Visual psychophysics on the Apple II: Getting started. Behavior Research Methods \& Instrumentation, 12, 614-626.

Julesz, B. (1971). Foundations of cyclopean perception. Chicago: University of Chicago Press.

MACKAY, D. M. (1965). Dynamic noise as a tool in visual research. Journal of General Psychology, 72, 181-197.

TYLER, C. W. (1974). Depth perception in disparity gratings. Nature, 251, 140-142. 